\title{
Evolución del traumatismo penetrante cardíaco operado
}

\author{
Roberto González L. ${ }^{1,2}$, Felipe Alarcón O. ${ }^{3}$, Alejandra Riquelme U. ${ }^{4}$, Rodrigo Reyes M. ${ }^{1,2}$, \\ Sebastián Barra M. ${ }^{4}$, Enrique Seguel S. ${ }^{1,2}$, Aleck Stockins L. ${ }^{1,2}$, Andrés Jadue T. ${ }^{1}$, \\ Diego Saldivia Z. ${ }^{4}$, Andrés Schaub C. ${ }^{4}$ y Emilio Alarcón C. ${ }^{1,2}$
}

\section{Penetrating cardiac injury: evolution of characteristics, severity and mortality} in surgery treated patients

Background: Penetrating cardiac injury (PCI) is a rare injury with high morbidity and mortality. Aim: To analyze the evolution of clinical and anatomical characteristics, severity, morbidity and mortality of patients operated on by PCI. Materials and Methods: Analytical study of patients surgically treated for PCI at the "Guillermo Grant Benavente" Regional Clinical Hospital, Concepción, Chile. Two periods were analyzed: January-1990 to December-2004 and January-2005 to December-2019. Sex, age, associated injuries, trauma agent and mechanism, pathophysiological behavior, anatomic location of the injury, classifications of cardiac trauma: Attar, Saadia and OIS-AAST, TSI (trauma severity indices): ISS, RTS-T and TRISS, morbidity and mortality were compared according to periods. Statistical analysis was performed with SPSS $25^{\circledR}$, the chi-square, Fisher exact and Mann-Whitney tests were used. A p value $<0.05$ was considered significant. Results: Total 235 PCI, 112 in the first period and 123 in the second. Stab as mechanism in $96(85.7 \%)$ and $104(84.6 \%)$ according to periods. An increase in associated extra thoracic injuries, cardiorespiratory arrest, and injury to the left location were observed in the second period. The cardiac trauma classifications and the TSI ISS, RTS-T and TRISS showed greater severity and probability of death in the second period patients. Mortality did not show differences: $14(12.5 \%)$ and $14(11.4 \%)$ according to periods, $p=0.792$. Discussion: In our series, patients treated with PCI have evolved towards a more severity profile in both, physiological and anatomical parameters. Mortality has been stable over time. Key words: heart injuries; cardiac tamponade; thoracic surgery; wounds, penetrating; trauma severity indices.

\section{Resumen}

Introducción: El traumatismo penetrante cardíaco (TPC) es una lesión poco frecuente y con alta morbilidad y mortalidad. Objetivo: Analizar la evolución de características clínicas, anatómicas, gravedad, morbilidad y mortalidad de pacientes operados por TPC. Materiales y Métodos: Estudio analítico de pacientes tratados quirúrgicamente por TPC en Hospital Clínico Regional "Dr. Guillermo Grant Benavente", Concepción, Chile. Se analizaron los periodos: enero-1990 a diciembre-2004 y enero-2005 a diciembre-2019. Se comparó: sexo, edad, lesiones asociadas, agente y mecanismo del traumatismo, comportamiento fisiopatológico, ubicación anatómica de la lesión, clasificaciones del traumatismo cardíaco Attar, Saadia y OIS-AAST, IGT (índices de gravedad del traumatismo): ISS, RTS-T y TRISS, morbilidad y mortalidad según periodos. Se realizó análisis estadístico con $S P S S 25^{\circledR}$, se utilizaron las pruebas chi-cuadrado, exacta de Fisher y Mann-Whitney. Se consideró significativo un valor $\mathrm{p}<0,05$. Resultados: Total 235 TPC, 112 en el primer periodo y 123 en el segundo. Mecanismo arma blanca en $96(85,7 \%)$ y 104 (84,6\%) según periodos. En el segundo periodo se observó un aumento de lesiones extratorácicas asociadas, paro cardiorrespiratorio y lesión de ubicación izquierda. Las clasificaciones del traumatismo cardíaco y los IGT ISS, RTS-T y TRISS mostraron mayor gravedad y probabilidad de muerte en los pacientes del segundo periodo. La mortalidad no mostró diferencias: $14(12,5 \%)$ y $14(11,4 \%)$ según periodos $(p=0,792)$. Discusión: En nuestra serie los pacientes tratados por TPC han evolucionado hacia un perfil de mayor gravedad tanto en parámetros fisiológicos como anatómicos. La mortalidad se ha mantenido estable a través del tiempo. Palabras clave: lesiones cardíacas; taponamiento cardíaco; cirugía torácica; heridas penetrantes; índices de gravedad del trauma.
${ }^{1}$ Centro Cardiovascular, Hospital Clínico Regional de Concepción "Dr. Guillermo Grant Benavente" Concepción, Chile. Departamento de Cirugía, Facultad de Medicina, Universidad de Concepción Concepción, Chile. ${ }^{3}$ Estudiante de Medicina, Facultad de Medicina, Universidad de Concepción Concepción, Chile. ${ }^{4}$ Servicio de Cirugía, Hospital Clínico Regional de Concepción "Dr. Guillermo Grant Benavente". Concepción, Chile.

Esta comunicación corresponde a uno de los análisis de la base de datos prospectiva de hospitalizaciones por traumatismo torácico iniciada el año 1981 en Concepción, Chile.

Recibido 2020-05-27 y aceptado 2020-07-03

Correspondencia a: Dr. Roberto González Lagos rgonzalezlagos@udec.cl 


\section{Introducción}

El traumatismo penetrante cardíaco (TPC) se puede definir como una lesión traumática por un objeto cortopunzante o penetrante, que origina una solución de continuidad de estructuras intrapericárdicas, incluyendo: cámaras cardíacas, arterias coronarias y/o grandes vasos intrapericárdicos. Es un grupo heterogéneo de lesiones que se presentan en un espectro clínico, fisiopatológico y terapéutico similar, caracterizándose por una alta morbilidad y mortalidad.

Se estima que aproximadamente un 60-90\% de los casos fallecen en el sitio del traumatismo o previo a la atención profesional prehospitalaria o intrahospitalaria ${ }^{1-5}$, aumentando considerablemente la supervivencia al recibir atención hospitalaria y accediendo a tratamiento definitivo. La mortalidad intrahospitalaria se describe aproximadamente entre un $12-70 \%$ según diversas series ${ }^{6}$.

Las variaciones en supervivencia pueden explicarse, entre otros motivos, por las características del hospital, servicios de traslado, protocolos de urgencia y equipos quirúrgicos, así como por variables sociodemográficas que determinan el agente y mecanismo más frecuente del traumatismo ${ }^{1,2,7}$.

Experiencias han demostrado que, en un mismo centro, las características de los TPC pueden presentar cambios al analizar distintos periodos ${ }^{1,2}$. En nuestro medio, existen escasas publicaciones que analicen las características del TPC.

Nuestro objetivo es analizar la evolución de características clínicas, anatómicas, gravedad, morbilidad y mortalidad de pacientes operados por TPC.

\section{Materiales y Métodos}

Estudio analítico longitudinal de pacientes tratados quirúrgicamente por TPC en el Hospital Clínico Regional "Dr. Guillermo Grant Benavente" de Concepción-Chile, entre enero de 1990 y diciembre de 2019.

La información se obtuvo de protocolos prospectivos de TPC realizados al ingreso, base de datos del equipo de Cirugía Cardiotorácica, documentos de urgencia, fichas clínicas, registros de pabellón y protocolos quirúrgicos.

Para analizar la evolución se dividieron los casos en periodos de 15 años, entre enero de 1990 a diciembre de 2004 y enero de 2005 a diciembre de 2019. Se comparan: sexo, edad, tratamiento prehospitalario, abordaje, lesiones asociadas, agente y mecanismo del traumatismo, comportamiento fisiopatológico, ubicación anatómica de la lesión, clasificaciones del traumatismo cardíaco, índices de gravedad del traumatismo (IGT), morbilidad y mortalidad según periodos.

Se agruparon los casos según clasificaciones del traumatismo cardíaco: Attar ${ }^{8}$, Saadia ${ }^{9}$ y OIS-AAST (Organ Injury Scale of American Association for the Surgery of Trauma $)^{10}$, y se calcularon los IGT: Injury Severity Score (ISS) ${ }^{11}$, Revised Trauma Score Triage $\left(\mathrm{RTS}_{\mathrm{T}}\right)^{12}$ y Trauma Injury Severity Score (TRISS) $^{13,14}$. Se consideró politraumatismo a los pacientes con lesiones en dos o más sistemas y con ISS $>16$ puntos $^{15}$. Para graficar la evolución según IGT se dividió la serie en periodos de seis años.

Se consideró atención profesional prehospitalaria a los pacientes trasladados por móviles del SAMU (Sistema de Atención Médica de Urgencia) o derivados desde otro centro.

Se registró el tiempo desde el traumatismo hasta el ingreso a nuestro hospital según la información entregada por el paciente $\mathrm{y} / \mathrm{o}$ acompañantes, y solo en los casos cuando estuvo disponible.

Para la descripción de regiones anatómicas intrapericárdicas lesionadas, se dividió en ubicación izquierda y derecha, según el lado de la cámara y/o vaso intrapericárdico lesionado.

Se clasificó el comportamiento fisiopatológico en taponamiento cardíaco o si presentó paro cardiorrespiratorio (PCR) en cualquier momento desde el ingreso al hospital hasta la cirugía.

Se tabularon datos en planilla Microsoft Excel ${ }^{\circledR}$ y se realizó análisis estadístico con programa $S P S S 25^{\circledR}$. Se utilizó la prueba chi-cuadrado o prueba exacta de Fisher para variables categóricas, según corresponda, y la prueba Mann-Whitney para variables continuas. Se consideró significativo $p<0,05$.

El estudio y análisis de las hospitalizaciones en pacientes con traumatismo torácico fue aprobado por el comité ético-científico de nuestra institución.

\section{Resultados}

Durante el periodo se operaron 238 traumatismos cardíacos, de los cuales $235(98,7 \%)$ fueron TPC y 3 $(1,3 \%)$ fueron por traumatismos cardíacos contusos. De los 235 TPC, se operaron 112 en el primer periodo y 123 en el segundo, observándose un aumento del $9,8 \%$ de casos. El sexo y edad según periodo se describen en la Tabla 1.

En relación a las variables prehospitalarias, el tiempo desde el traumatismo al ingreso se registró en $210(89,4 \%)$ casos y no mostró diferencias significativas entre periodos. Se registró un aumento 
Tabla 1. Sexo, edad, atención profesional prehospitalaria y tiempo de traslado de pacientes tratados por traumatismo penetrante cardíaco, según periodo

\begin{tabular}{|lccc|}
\hline & $\begin{array}{c}\mathbf{1 9 9 0 - 2 0 0 4} \\
(\mathbf{n = 1 1 2})\end{array}$ & $\begin{array}{c}\mathbf{2 0 0 5 - 2 0 1 9} \\
(\mathbf{n = 1 2 3})\end{array}$ & $\mathbf{p}$ \\
\hline $\begin{array}{l}\text { Sexo } \\
\text { Hombre }\end{array}$ & $104(92,9 \%)$ & $120(97,6 \%)$ & 0,088 \\
$\quad$ Mujer & $8(7,1 \%)$ & $3(2,4 \%)$ & \\
Edad & $30,5 \pm 13,5$ & $29,7 \pm 12,9$ & 0,686 \\
$\quad \begin{array}{l}\text { Promedio } \\
\text { Mediana }\end{array}$ & 26,5 & 27 & 0,664 \\
Tiempo desde el traumatismo hasta el ingreso (min) & $96,4 \pm 149,8$ & $78,7 \pm 158,1$ & $\mathbf{0 , 0 0 9 *}$ \\
\hline Atención profesional prehospitalaria & $10(8,9 \%)$ & $26(21,1 \%)$ & \\
\hline
\end{tabular}

*Estadísticamente significativo.

significativo de la atención prehospitalaria, desde 10 $(8,9 \%)$ a $26(21,1 \%)$ pacientes (Tabla 1$)$.

Si bien la lesión torácica aislada predominó en ambos periodos, se observó un aumento significativo de lesiones extratorácicas asociadas, de 7 (6,3\%) a $19(15,4 \%)$ pacientes. El agente del traumatismo más frecuente fue el arma blanca en $96(85,7 \%)$ y $104(84,6 \%)$ pacientes, según periodos (Tabla 2).

En cuanto al comportamiento fisiopatológico, se observó un incremento significativo del PCR; desde $4(3,6 \%)$ a $19(15,4 \%)$ casos. El taponamiento cardíaco disminuyó significativamente; desde 95 $(86,4 \%)$ a $84(68,9 \%)$ casos, según periodos (Figura $1)$.

La ubicación anatómica de la lesión fue más frecuente en territorio derecho en ambos periodos, sin embargo, la lesión del territorio izquierdo se incrementó significativamente desde $36(32,1 \%)$ a $55(44,7 \%)$ casos. Se observó una reducción significativa en lesiones del ventrículo derecho, desde 65
Tabla 2. Tipo, agente y mecanismo del traumatismo penetrante cardíaco, según periodo

\begin{tabular}{|lrrc|}
\hline & $\begin{array}{c}\mathbf{1 9 9 0 - 2 0 0 4} \\
(\mathbf{n = 1 1 2})\end{array}$ & $\begin{array}{r}\mathbf{2 0 0 5 - 2 0 1 9} \\
(\mathbf{n}=\mathbf{1 2 3})\end{array}$ & $\mathbf{p}$ \\
Tipo de TPC & & & \\
$\quad$ Lesión torácica aislada & $105(93,7 \%)$ & $104(84,6 \%)$ & $\mathbf{0 , 0 2 5}$ \\
Lesión extratorácica asociada & $7(6,3 \%)$ & $19(15,4 \%)$ & \\
$\quad$ Politraumatismo & $7(6,3 \%)$ & $12(9,8 \%)$ & 0,325 \\
Agente & & & \\
Arma blanca & $96(85,7 \%)$ & $104(84,6 \%)$ & 0,803 \\
Arma de fuego & $10(8,9 \%)$ & $12(9,8 \%)$ & 0,828 \\
Otro & $6(5,4 \%)$ & $7(5,7 \%)$ & 0,711 \\
Mecanismo & & & \\
Agresión & $103(91,9 \%)$ & $113(91,9 \%)$ & 0,979 \\
Iatrogénico & $5(4,5 \%)$ & $7(5,7 \%)$ & 0,670 \\
Autoagresión & $3(2,7 \%)$ & $2(1,6 \%)$ & 0,577 \\
Accidental & $1(0,9 \%)$ & $1(0,8 \%)$ & 0,947 \\
\hline
\end{tabular}

TPC: Traumatismo penetrante cardíaco; *Estadísticamente significativo.

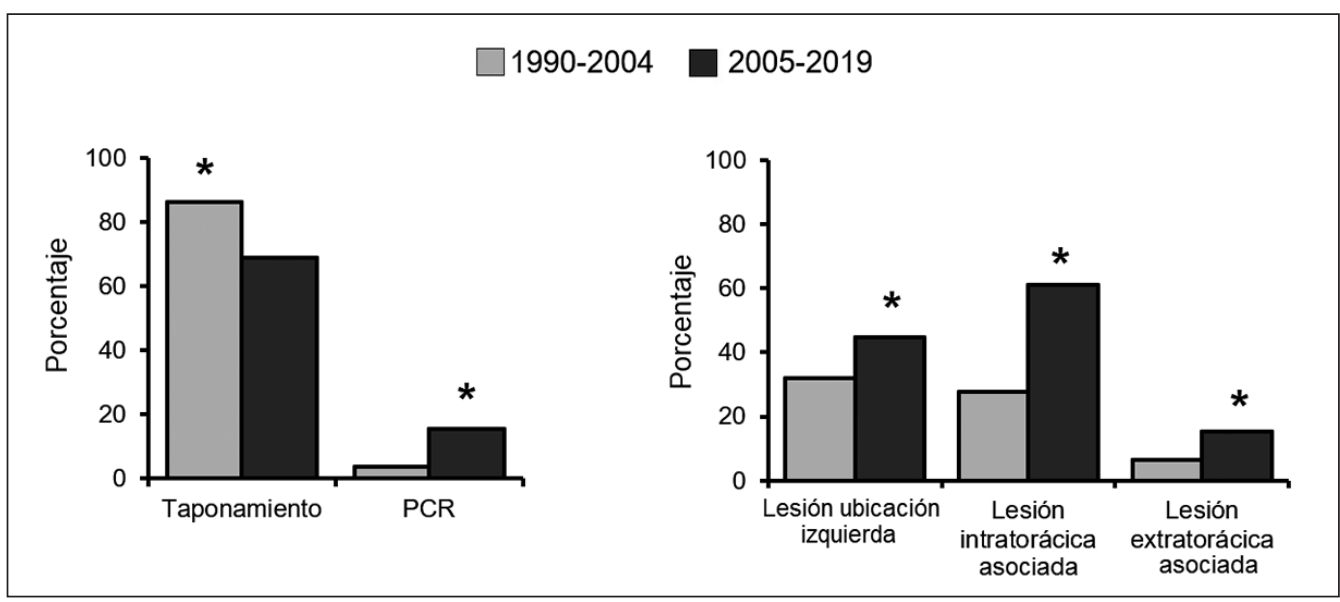

Rev. Cir. 2021;73(4):401-409
Figura 1. Comportamiento fisiopatológico y lesiones anatómicas con diferencias significativas entre periodos en pacientes tratados por traumatismo penetrante cardíaco. PCR: Paro cardiorespiratorio; *Estadísticamente significativo 


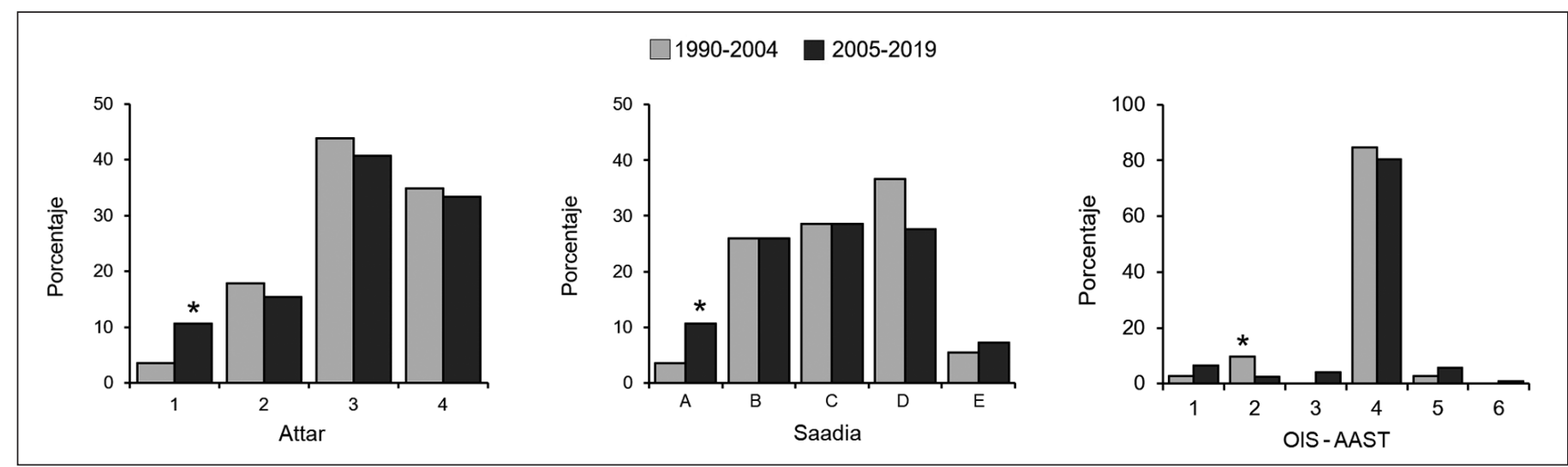

Figura 2. Distribución porcentual según clasificaciones del traumatismo cardíaco. OIS-AAST: Organ Injury Scale of American Association for the Surgery of Trauma; *Estadísticamente significativo

Tabla 3. Comportamiento fisiopátologico y lesiones y/o hallazgos anatómicos cardíacos e intratorácicos en pacientes tratados por traumatismo penetrante cardíaco, según periodo

\begin{tabular}{|lrrr|}
\hline & $\begin{array}{c}\mathbf{1 9 9 0 - 2 0 0 4} \\
(\mathbf{n = 1 1 2})\end{array}$ & $\begin{array}{c}\mathbf{2 0 0 5 - 2 0 1 9} \\
(\mathbf{n = 1 2 3})\end{array}$ & $\mathbf{p}$ \\
\hline $\begin{array}{l}\text { Comportamiento fisiopatológico } \\
\text { Taponamiento cardiaco }\end{array}$ & $95(86,4 \%)$ & $84(68,9 \%)$ & $\mathbf{0 , 0 0 2 *}$ \\
Paro cardiorrespiratorio & $4(3,6 \%)$ & $19(15,4 \%)$ & $<\mathbf{0 , 0 0 1 *}$ \\
Ubicación de la lesión & & & \\
Izquierda & $36(32,1 \%)$ & $55(44,7 \%)$ & $\mathbf{0 , 0 3 4} *$ \\
Derecha & $76(67,9 \%)$ & $68(55,3 \%)$ & \\
Lesión arteria coronaria & $5(4,5 \%)$ & $9(7,3 \%)$ & 0,356 \\
\hline Lesión intratorácica asociada** & $31(27,7 \%)$ & $75(61,0 \%)$ & $<\mathbf{0 , 0 0 1 *}$ \\
\hline
\end{tabular}

*Estadísticamente significativo; **Lesiones y/o hallazgos.

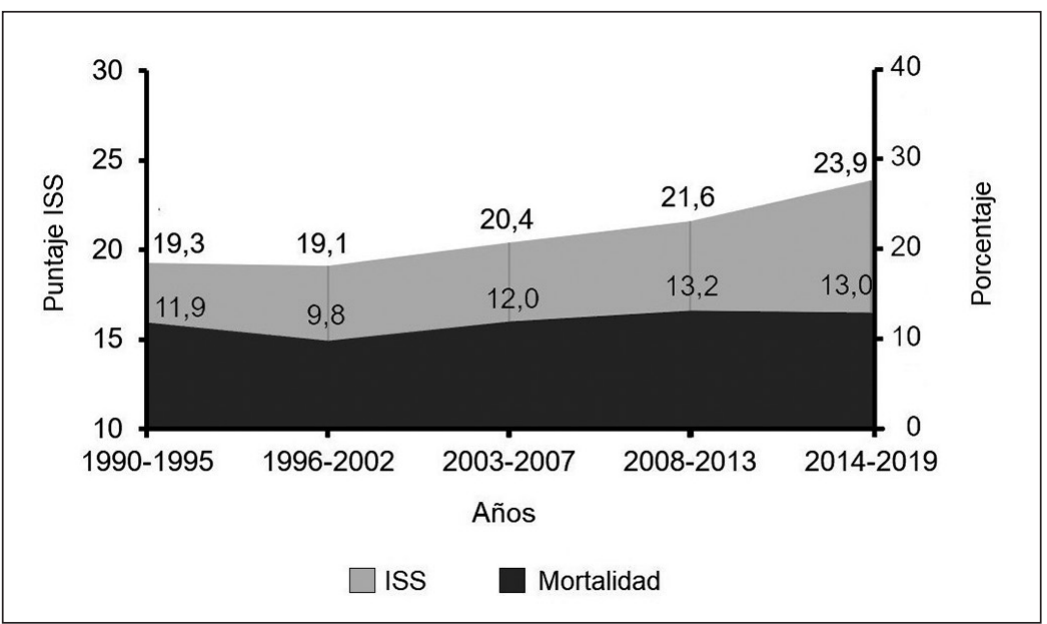

Figura 3. Evolución del puntaje promedio de Injury Severity Score y la mortalidad en pacientes tratados por traumatismo penetrante cardíaco. ISS: Injury Severity Score.
$(58,0 \%)$ a $52(42,3 \%)$ casos. La lesión de grandes vasos intrapericárdicos más frecuente fue la aórtica en $11(5 \%)$ casos. Las lesiones intratorácicas asociadas aumentaron significativamente desde $31(27,7 \%)$ a $75(61,0 \%)$ pacientes (Tabla 3 y Figura 1$)$.

En relación a las clasificaciones del traumatismo cardíaco, se observaron diferencias significativas en pacientes de categorías de extrema gravedad. Tanto el grupo Attar 1 como Saadia A aumentaron significativamente; desde $4(3,6 \%)$ a $13(10,6 \%)$ pacientes. Por otro lado, el número de casos en la categoría OIS-AAST 2 (menor gravedad) disminuyó significativamente de $11(9,8 \%)$ a $3(2,4 \%)$ casos (Figura 2). En los IGT, se observó aumento significativo de la gravedad según ISS, RTS-T y TRISS, denotando mayor gravedad anatómica, peores parámetros fisiológicos y peor pronóstico de supervivencia (Tabla 4 y Figuras 3-5). No se encontraron diferencias significativas en morbilidad y mortalidad global, sin embargo, se observó un aumento en la mortalidad intraoperatoria $(\mathrm{p}=0,008)$ (Tabla 5).

En relación al abordaje quirúrgico, en el primer periodo, en $64(57,1 \%)$ y $48(42,9 \%)$ pacientes se realizó esternotomía y toracotomía, respectivamente, mientras que durante el segundo periodo se observó un aumento de las esternotomías, en 106 (86,2\%) pacientes y una disminución significativa de las toracotomías, en $17(13,8 \%)$ pacientes, $\mathrm{p}<0,001$ (Tabla 5).

En el subgrupo de pacientes con lesión de cámaras cardíacas aisladas (con exclusión de lesiones de grandes vasos intrapericárdicos y lesiones intra y/o extratorácicas asociadas) se observó un aumento en la gravedad según ISS, RTS-T y de la mortalidad esperada (TRISS). La mortalidad se mantuvo sin diferencias significativas $(\mathrm{p}=0,566)$, (Tabla 4). 
Tabla 4. Clasificaciones del traumatismo cardíaco e índices de gravedad del traumatismo, según periodo

\begin{tabular}{|c|c|c|c|}
\hline Clasificaciones del TPC & $\begin{array}{c}1990-2004 \\
(n=112)\end{array}$ & $\begin{array}{c}2005-2019 \\
(n=123)\end{array}$ & $\mathbf{p}$ \\
\hline \multicolumn{4}{|l|}{ Attar } \\
\hline 1 & $4(3,6 \%)$ & $13(10,6 \%)$ & $0,039 *$ \\
\hline 2 & $20(17,9 \%)$ & $19(15,4 \%)$ & 0,620 \\
\hline 3 & $49(43,7 \%)$ & $50(40,7 \%)$ & 0,631 \\
\hline 4 & $39(34,8 \%)$ & $41(33,3 \%)$ & 0,810 \\
\hline \multicolumn{4}{|l|}{ Saadia } \\
\hline A & $4(3,6 \%)$ & $13(10,6 \%)$ & $0,039 *$ \\
\hline B & $29(25,9 \%)$ & $32(26,0 \%)$ & 0,983 \\
\hline $\mathrm{C}$ & $32(28,5 \%)$ & $35(28,5 \%)$ & 0,984 \\
\hline $\mathrm{D}$ & $41(36,6 \%)$ & $34(27,6 \%)$ & 0,141 \\
\hline $\mathrm{E}$ & $6(5,4 \%)$ & $9(7,3 \%)$ & 0,539 \\
\hline \multicolumn{4}{|l|}{ OIS-AAST } \\
\hline 1 & $3(2,7 \%)$ & $8(6,5 \%)$ & 0,166 \\
\hline 2 & $11(9,8 \%)$ & $3(2,4 \%)$ & $0,017 *$ \\
\hline 3 & $0(0,0 \%)$ & $5(4,1 \%)$ & 0,061 \\
\hline 4 & $95(84,8 \%)$ & $99(80,5 \%)$ & 0,382 \\
\hline 5 & $3(2,7 \%)$ & $7(5,7 \%)$ & 0,339 \\
\hline 6 & $0(0,0 \%)$ & $1(0,8 \%)$ & 1,000 \\
\hline \multicolumn{4}{|l|}{ IGT global } \\
\hline Injury Severity Score & $19,2 \pm 7,3$ & $22,5 \pm 8,2$ & $<0,001 *$ \\
\hline Revised Trauma Score Triage & $9,5 \pm 3,3$ & $8,5 \pm 4,0$ & $\mathbf{0 , 0 2 5} *$ \\
\hline Trauma Injury Severity Score & 21,7 & 31,4 & $0,007 *$ \\
\hline Mortalidad & $14(12,5 \%)$ & $14(11,4 \%)$ & 0,792 \\
\hline IGT en subgrupo con lesión aislada de cámara & $95(84,8 \%)$ & $87(70,7 \%)$ & \\
\hline Injury Severity Score & $18,1 \pm 4,5$ & $21,9 \pm 5,0$ & $<0,001 *$ \\
\hline Revised Trauma Score Triage & $9,5 \pm 3,3$ & $8,8 \pm 3,8$ & 0,062 \\
\hline Trauma Injury Severity Score & 21,3 & 28,3 & $0,024 *$ \\
\hline Mortalidad & $10(10,5 \%)$ & $7(8,0 \%)$ & 0,566 \\
\hline
\end{tabular}

TPC: Traumatismo penetrante cardíaco; OIS-AAST: Organ Injury Scale of American Association for the Surgery of Trauma; IGT: Índice de gravedad del traumatismo *: Estadísticamente significativo.

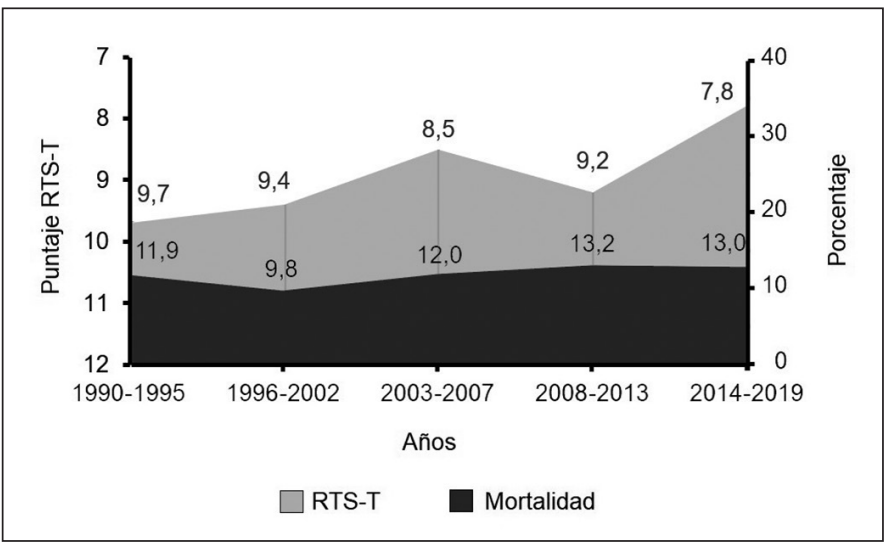

Figura 4. Evolución del puntaje promedio de Revised Trauma Score Triage y mortalidad en pacientes tratados por traumatismo penetrante cardíaco. RTS-T: Revised Trauma Score Triage.

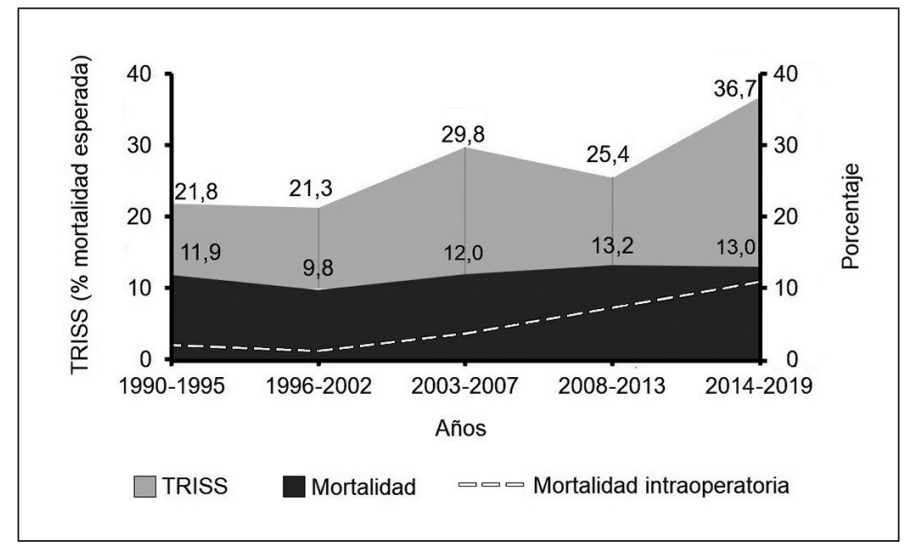

Figura 5. Evolución de la mortalidad esperada según TRISS y mortalidad observada en pacientes tratados por traumatismo penetrante cardíaco. TRISS: Trauma Injury Severity Score. 
Tabla 5. Abordaje, cirugía extratorácicas, transfusiones, morbilidad, reoperación, estadía hospitalaria y mortalidad de pacientes tratados por traumatismo penetrante cardíaco según periodos

\begin{tabular}{|lccc|}
\hline & $\begin{array}{c}\mathbf{1 9 9 0 - 2 0 0 4} \\
(\mathbf{n}=\mathbf{1 1 2})\end{array}$ & $\begin{array}{c}\mathbf{2 0 0 5 - 2 0 1 9} \\
(\mathbf{n = 1 2 3})\end{array}$ & $\mathbf{p}$ \\
Abordaje & & & \\
$\quad$ Esternotomía & $64(57,1 \%)$ & $106(86,2 \%)$ & $<\mathbf{0 , 0 0 1 *}$ \\
$\quad$ Toracotomía & $48(42,9 \%)$ & $17(13,8 \%)$ & \\
Cirugía extratorácica & $5(4,5 \%)$ & $12(9,8 \%)$ & 0,118 \\
Transfusión & $34(30,4 \%)$ & $45(36,6 \%)$ & 0,313 \\
$\quad$ Unidades por paciente transfundido & & & \\
$\quad$ Promedio & $6,4 \pm 6,7$ & $11,6 \pm 11,0$ & $<\mathbf{0 , 0 0 1 *}$ \\
$\quad$ Mediana & 5 & 8 & \\
Morbilidad & $33(29,5 \%)$ & $40(32,5 \%)$ & 0,613 \\
$\quad$ Deterioro neurológico & $8(7,1 \%)$ & $5(4,1 \%)$ & 0,303 \\
Reoperación & $14(12,5 \%)$ & $11(8,9 \%)$ & 0,377 \\
Estadía hospitalaria (días) & & & \\
$\quad$ Promedio & $10,0 \pm 15,4$ & $8,5 \pm 6,9$ & 0,571 \\
$\quad$ Mediana & 7 & 6 & \\
Hospitalización en UCI & $58(51,8 \%)$ & $91(74,0 \%)$ & $<\mathbf{0 , 0 0 1 *}$ \\
Mortalidad global & $14(12,5 \%)$ & $14(11,4 \%)$ & 0,792 \\
Intraoperatoria & $3(21,4 \%)$ & $10(71,4 \%)$ & $\mathbf{0 , 0 0 8 *}$ \\
Posoperatoria & $11(78,6 \%)$ & $4(28,6 \%)$ & \\
\hline
\end{tabular}

UCI: Unidad de cuidados intensivos. *Estadísticamente significativo.

\section{Discusión}

En nuestra serie ha aumentado la gravedad de los pacientes tratados por TPC. Se observó un incremento del PCR, lesiones intra y extratorácicas asociadas y lesión en la ubicación izquierda, con el consecuente aumento de la proporción de las categorías de mayor gravedad de clasificaciones del traumatismo cardíaco y de los IGT. Sin embargo, no encontramos diferencias significativas en la mortalidad.

El tiempo transcurrido desde el traumatismo hasta la atención profesional y el tratamiento definitivo se considera determinante en el pronóstico, no obstante, esta información no siempre es reportada u objetivamente documentada, es por esto que frecuentemente se utiliza el tiempo transcurrido entre la llegada de los servicios de traslado al lugar del traumatismo y su llegada a las unidades de urgencias, siendo este dato no representativo del tiempo de evolución real del traumatismo ${ }^{2,16}$. En nuestra serie, el tiempo desde el traumatismo hasta el ingreso hospitalario (cuando fue posible obtenerlo) presentó diferencias entre períodos, aunque sin alcanzar significación estadística. Esta información debe evaluarse con cautela por ser un dato no siempre disponible ya sea porque el paciente o quienes lo acompañan no desean aportar información, porque entregan información imprecisa o porque el paciente es encontrado en la vía pública bajo los efectos del alcohol y/o drogas. Por otro lado, existen casos que evolucionan sin deterioro hemodinámico inicial, consultando días posteriores al traumatismo y en quienes se diagnostica un taponamiento cardíaco tardío ${ }^{17,18}$.

A diferencia del tiempo desde el traumatismo al ingreso, la atención profesional prehospitalaria es una variable siempre consignada. En nuestra serie se observó un incremento significativo en la atención prehospitalaria, sin embargo, este porcentaje es bajo en relación a los pacientes que acuden al hospital trasladados por terceros o incluso por sus propios medios (aproximadamente $80 \%$ en nuestra serie). Este aspecto es característico de los traumatismos penetrantes por arma blanca y difiere considerablemente de los traumatismos contusos graves tales como el politraumatismo en el contexto de accidentes de tránsito, quienes en su mayoría requieren imperativamente asistencia de un sistema de traslado. En nuestro medio, la atención prehospitalaria es realizada principalmente por el sistema SAMU, el cual fue implementado en nuestro hospital en noviembre de 1998.

El comportamiento fisiopatológico del TPC puede clasificarse en términos prácticos en al menos cuatro grandes grupos: sin deterioro hemodinámico, taponamiento cardíaco, PCR y shock. El taponamiento cardíaco se describe aproximadamente entre el $55-75 \%{ }^{19-21}$ de los casos. Tradicionalmente se considera un factor protector de mortalidad a expensas de su capacidad de limitar el sangrado y evitar la exanguinación ${ }^{20,22,23}$, sin embargo, existe controversia en la literatura reportándose publicaciones que no encontraron esta asociación ${ }^{5,24}$. Cabe destacar que las series que encontraron un efecto protector se caracterizaron porque son mayoritariamente por arma blanca, en cambio, las series en que no se encontró dicho efecto están compuestas principalmente por arma de fuego. Por ello, es posible que el mayor número de lesiones y la gravedad que caracteriza al grupo con TPC por arma de fuego sean responsables de disminuir el efecto protector del taponamiento cardíaco. En nuestra serie se observó una disminución porcentual de los pacientes con taponamiento cardíaco.

El PCR es uno de los mayores predictores de mortalidad en TPC, incluida nuestra experien$\mathrm{cia}^{25}$, y se le atribuye una mortalidad entre un 80 - 
$100 \%{ }^{16,20,26,27}$. Los pacientes que presentaron PCR aumentaron significativamente, lo que creemos refleja una mejora en la capacidad de rescate y de reanimación de este subgrupo, pese a su gravedad.

Por otro lado, el TPC puede presentarse como un hemotórax masivo en ausencia de taponamiento cardíaco progresivo, secundario a una lesión del pericardio hacia la cavidad pleural que permite un vaciamiento hacia ella. Estos pacientes se caracterizan por una evolución progresiva hacia el shock hipovolémico y a la exanguinación ${ }^{3,28}$. En una serie de nuestro grupo, el TPC fue responsable del 15\% de los hemotórax masivos y se observó una mortalidad del $39 \%$ en este subgrupo ${ }^{29}$.

Durante las últimas décadas, en la literatura internacional, el agente del traumatismo ha experimentado una transición hacia un aumento del arma de fuego como agente causal ${ }^{1,2,6}$. El TPC secundario a armas de fuego se relaciona con traumatismos más graves, compromiso de múltiples cámaras, sangrado irregular en el espacio intrapericárdico, lesiones extracardíacas asociadas y mayor mortalidad, constituyendo uno de los principales factores pronósticos $^{1-4,30-33}$. En nuestra serie, la proporción del TPC por armas de fuego aumentó, aunque no alcanzó significancia estadística y se mantuvo inferior al $10 \%$ de los casos, lo que podría explicar, en parte, la baja mortalidad de nuestra serie.

Se observó un aumento en las lesiones extratorácicas principalmente a expensas de lesiones abdominales, lo que concuerda con otras series ${ }^{16,25}$. Los traumatismos penetrantes toracoabdominales per sé son consideradas lesiones con elevada mortalidad, descrita entre un $13-41 \%{ }^{34-37}$ y se observan más frecuentemente en traumatismos por arma de fuego ${ }^{30,34}$.

La posición anatómica del corazón en relación a la pared del tórax determina que por su posición anterior, el ventrículo derecho sea el más frecuentemente lesionado, alcanzando hasta el $60 \%$ de los $\operatorname{casos}^{1,2,19,21,38-40}$. Las lesiones del territorio derecho del corazón se relacionan con un mejor pronóstico en ciertas series ${ }^{40,41}$, lo que se explicaría debido a sus menores presiones sanguíneas, provocando un menor sangrado y cuadros más estables en comparación con la lesión del territorio izquierdo. El aumento de los pacientes con lesiones del territorio izquierdo es otro factor que creemos aumenta la gravedad de los pacientes pertenecientes al último periodo.

En nuestra serie, tanto las clasificaciones del traumatismo cardíaco como los IGT demostraron una mayor gravedad en el segundo periodo. Las clasificaciones Attar y Saadia utilizan parámetros fisiológicos y del comportamiento fisiopatológico del TPC y mostraron un aumento en los pacientes pertenecientes a los grupos de máxima gravedad (Attar 1 y Saadia A) que fundamentalmente incluyen pacientes sin signos vitales al ingreso. La clasificación OIS-AAST, por otro lado, se centra principalmente en la anatomía de la lesión y mostró una disminución de los pacientes con lesiones de menor gravedad correspondientes a lesiones tangenciales del miocardio y sin taponamiento cardíaco. Los IGT, a pesar de no ser específicos del TPC, utilizan variables fisiológicas y anatómicas tomando en cuenta, además, lesiones extratorácicas para estimar el pronóstico. En los tres IGT utilizados fue posible observar un aumento de la gravedad de los casos a través del tiempo.

En nuestro análisis se incluyeron en la ubicación izquierda-derecha sus respectivos grandes vasos intrapericárdicos, lesiones que en concomitancia con lesiones de cámaras cardíacas han demostrado un peor pronóstico ${ }^{30,40}$, es por ello que realizamos un análisis del subgrupo con lesión exclusiva de cámara cardíaca y sin otras lesiones intra y/o extratorácicas a modo de comparar un grupo característico y homogéneo del TPC. Nuestros resultados son comparables al análisis global del grupo, evidenciando un aumento en la gravedad del traumatismo dado por los IGT y con una mortalidad sin cambios significativos entre los periodos. Este análisis demuestra que, además del aumento de lesiones asociadas, se observó mayor gravedad de la lesión cardíaca en el segundo periodo, lo que podría explicar el aumento de la proporción de la mortalidad intraoperatoria.

Morse y cols ${ }^{1}$, en un análisis en tres periodos encontraron un aumento de la mortalidad, atribuyendolo a la mejora en los sistemas de traslado que posibilitan que más pacientes "moribundos" accedan a tratamiento. Además, observaron un aumento significativo de las lesiones por arma de fuego, lo que contribuyó a un peor pronóstico.

Pereira y cols ${ }^{2}$, en un análisis en dos periodos reportaron, a diferencia de nuestros hallazgos, mejores parámetros fisiológicos y una mayor supervivencia en el segundo periodo, infiriendo que dicha mejora puede ser provocada por la optimización de los servicios prehospitalarios. La disparidad de esta serie frente a nuestros resultados, creemos responden a diferencias en las poblaciones seleccionadas, debido a que estos autores reportan una disminución de los niveles de violencia civil y del TPC en la región de estudio.

En nuestra serie, creemos que los cambios observados son consecuencia de un avance en los cuidados pre e intrahospitalarios posibilitando el ingreso y tratamiento de pacientes cada vez más graves. 
Dentro de estos avances, la implementación del sistema SAMU contribuyó al aumento de la atención prehospitalaria y posibilitó la atención hospitalaria de pacientes que previamente fallecían en el lugar del traumatismo. Por otro lado, la acumulación de experiencia de los equipos quirúrgicos en el tratamiento de esta lesión otorga un mejor pronóstico intrahospitalario a estos pacientes. Además, a diferencia de otras publicaciones, la proporción del arma de fuego como agente del traumatismo cardíaco en nuestra serie contribuye a mantener una mortalidad estable a través del tiempo.

En conclusión, en nuestra serie los pacientes tratados por TPC han evolucionado hacia un perfil de mayor gravedad tanto en parámetros fisiológicos como anatómicos. Estos cambios han tributado en las clasificaciones del traumatismo cardíaco e IGT observándose un aumento de la gravedad, sin cambios en la mortalidad.

\section{Responsabilidades éticas}

Protección de personas y animales. Los autores declaran que para esta investigación no se han realizado experimentos en seres humanos ni en animales.

Confidencialidad de los datos. Los autores declaran que en este artículo no aparecen datos de pacientes.

Conflictos de interés: no hay.

\section{Bibliografía}

1. Morse B, Mina M, Carr J, Jhunjhunwala R, Dente C, Zink J, et al. Penetrating cardiac injuries: A 36-year perspective at an urban, Level I trauma center. J Trauma Acute Care Surg. 2016;81:623-31.

2. Pereira BMT, Baltazar V, Rodríguez T, Pinheiro M, Petrucci O, Pereira G. Penetrating cardiac trauma: $20-y$ experience from a university teaching hospital. J Surg Res. 2013;183:792-7.

3. Kang N, Hsee L, Rizoli S, Alison P. Penetrating cardiac injury: overcoming the limits set by Nature. Injury 2009;40:91927.

4. Asensio JA, Berne JD, Demetriades D, Chan L, Murray J, Falabella A, et al. One hundred five penetrating cardiac injuries: a 2-year prospective evaluation. J Trauma 1998;44:1073-82.

5. Araújo AO, Westphal FL, Lima LC, Correia JO, Gomes PH, Costa EN, et al. Fatal cardiac trauma in the city of Manaus, Amazonas state, Brazil. Rev Col Bras Cir. 2018;45:e1888.

6. Campbell NC, Thomson SR, Muckart DJ, Meumann CM, Van Middelkoop I, Botha JB. Review of 1198 cases of penetrating cardiac trauma. Brit J Surg. 1997;84:173740.

7. Bellister S, Dennis B, Guillamondegui O. Blunt and Penetrating Cardiac Trauma. Surg Clin N Am. 2017;97:1065-76.

8. Attar S, Suter Ch, Hankins J, Sequeira
A, McLaughlin J. Penetrating Cardiac Injuries. Ann Thorac Surg. 1991;51:711-6.

9. Saadia R, Levy RD, Degiannis E, Velmahos GC. Penetrating cardiac injuries: clinical classification and management strategy. Brit J Surg. 1994:81:1572-5.

10. Moore EE, Malangoni MA, Cogbill TH, Shackford SR, Champion HR, Jurkovich GJ, et al. Organ injury scaling. IV: Thoracic vascular, lung, cardiac, and diaphragm. Trauma 1994;36:299-300.

11. Baker SP, O’Neill B, Haddon W, Long W. The Injury Severity Score: a method for describing patients with multiple injuries and evaluating emergency care. J Trauma 1974;14:187-96.

12. Champion H, Sacco W, Copes W, Gann D, Gennarelli T, Flanagan M. A revision of the Trauma Score. J Trauma 1989;29:6239.

13. Boyd C, Tolson M, Copes W. Evaluating trauma care: the TRISS method. Trauma Score and Injury Severity Score. J Trauma 1987;27:370-8.

14. Freixinet J, Beltrán J, Rodríguez P, Juliá G, Hussein M, Gil R, et al. Indicators of Severity in Chest Trauma. Arch Bronconeumol. 2008:44:257-62.

15. Mica L, Rufibach K, Keel M, Trentz O. The risk of early mortality of polytrauma patients associated to ISS, NISS, APACHE II values and prothrombin time. J Trauma Manag Outcomes 2013;7:6.

16. Asensio J, Murray J, Demetriades D,
Berne J, Cornwell E, Velmahos G, et al. Penetrating Cardiac Injuries: A Prospective Study of Variables Predicting Outcomes. J Am Coll Surg. 1998;186:2434.

17. Rendón F, Gómez-Danés L, Castro M. Delayed Cardiac Tamponade after Penetrating Thoracic Trauma. Asian Cardiovasc Thorac Ann. 2004;12:139-42.

18. Mechem C, Alam G. Delayed cardiac tamponade in a patient with penetrating chest trauma. J Emerg Med. 1997; 15:31-3.

19. Bamous M, Abdessemad A, Tadili J, Kettani A, Faroudy M. Evaluation of penetrating cardiac stab wounds. Scand J Trauma Resus. 2016:24:6.

20. Mina M, Jhunjhunwala R, Gelbard R, Dougherty S, Carr J, Dente C, et al. Factors Affecting Mortality After Penetrating Cardiac Injuries 10-year Experience at Urban Level I Trauma Center. Am J Surg. 2017;213:1109-15.

21. Janati M, Bolandparvaz S, Salaminia S, Ghoddusi H, Sabet B, Kojuri J. Outcome of penetrating cardiac injuries in southern Iran, Shiraz. Chin J Traumatol. 2013;16:89-93.

22. Moreno C, Moore EE, Majure JA, Hopeman AR. Pericardial Tamponade: A Critical Determinant for Survival Following Penetrating Cardiac Wounds. J Trauma 1986;26:821-5.

23. Degiannis E, Loogna P, Doll D, Bonanno F, Bowley D, Smith M, et al. 
Penetrating Cardiac Injuries: Recent Experience in South Africa. World J Surg. 2006;30:1258-64

24. Buckman RF, Badellino MM, Mauro LH, Asensio JA, Caputo C, Gass J. Penetrating Cardiac Wounds: Prospective Study of Factors Influencing Initial Resuscitation. J Trauma 1993;34:717-25

25. González R, Riquelme A, Fuentes A, Canales J, Seguel E, Stockins, et al. Traumatismo penetrante cardíaco: caracterización, resultados inmediatos y variables asociadas a morbilidad y mortalidad en pacientes operados. Rev Cir. 2019;71:245-52.

26. Seamon MJ, Shiroff AM, Franco M, Stawicki SP, Molina EJ, Gaughan JP, et al. Emergency department thoracotomy for penetrating injuries of the heart and great vessels: an appraisal of 283 consecutive cases from two urban trauma centers. J Trauma 2009;67:1250-7.

27. Rhee PM, Acosta J, Bridgeman A, Wang D, Rich N. Survival after emergency department thoracotomy: review of published data from the past 25 years. J Am Coll Surg. 2000;190:288-98.

28. Connelly TM, Kolcow W, Veerasingam D, DaCosta M. A severe penetrating cardiac injury in the absence of cardiac tamponade. Interact Cardiovasc Thorac Surg. 2017;24:286-7.
29. González R, Riquelme A, Toloza C, Reyes R, Seguel E, Stockins A, et al. Hemotórax masivo por traumatismo torácico en pacientes tratados quirúrgicamente. Rev Cir. 2020;72: [In Press].

30. Asensio JA, Ogun OA, Petrone P, Perez-Alonso AJ, Wagner M, Bertellotti $\mathrm{R}$, et al. Penetrating cardiac injuries: predictive model for outcomes based on 2016 patients from the National Trauma Data Bank. Eur J Trauma Emerg Surg. 2018;44:835-41.

31. Rhee PM, Foy H, Kaufmann C, Areola C, Boyle E, Maier RV, et al. Penetrating cardiac injuries: a population-based study. J Trauma 1998;45:366-70.

32. Thourani VH, Feliciano DV, Cooper WA, Brady KM, Adams AB, Rozycki GS, et al. Penetrating cardiac trauma at an urban trauma center: a 22-year perspective. Am Surg. 1999;65:811-6.

33. González R, Riquelme A, Ávalos M, Reyes R, Seguel E, Stockins A, et al. Traumatismo torácico por arma de fuego. Rev Cir. 2020;72: [In press].

34. Asensio JA, Arroyo H, Veloz W, Forno W, Gambaro E, Roldan GA, et al. Penetrating Thoracoabdominal Injuries: Ongoing Dilemma Which Cavity and When? World J Surg. 2001;26:539-43.

35. Hirshberg A, Wall MJ Jr., Allen
MK, Mattox KL. Double jeopardy: thoracoabdominal injuries requiring surgical intervention in both chest and abdomen. J Trauma 1995;39:225-9.

36. González R, Riquelme A, Fuentes A, Saldías R, Reyes R, Seguel E, et al. Traumatismo torácico: caracterización de hospitalizaciones durante tres décadas. Rev Med Chile 2018;146:196-205.

37. González R, Riquelme A, Toloza C, Reyes R, Seguel E, Stockins A, et al. Traumatismo torácico por arma blanca. Rev Cir. 2020;72:137-43.

38. Karrel R, Shaffer MA, Franaszek JB. Emergency Diagnosis, Resuscitation, and Treatment of Acute Penetrating Cardiac Trauma. Ann Emerg Med. 1982;11:50417.

39. Asensio JA, Garcia-Nunez LM, Petrone P. In: Feliciano DV, Mattox KL, Moore EE, editors. Trauma to the Heart. Trauma 6th ed., New York: McGraw Hill; 2008;56988.

40. Lustenberger $\mathrm{T}$, Talving $\mathrm{P}$, Lam L, Inaba K, Mohseni S, Ann J, et al. Penetrating cardiac trauma in adolescents: A rare injury with excessive mortality. J Pediatr Surg. 2013;48:745-9.

41. Demetriades D, van der Veen BW. Penetrating injuries of the heart: experience over two years in South Africa. J Trauma 1983;23:1034-41. 